\title{
Capacidade reprodutiva de Bothrops jararaca (Serpentes, Viperidae)
}

\author{
Thélia Rosana Forte Janeiro-Cinquini
}

Laboratório de Herpetologia, Instituto Butantan, Av. Vital Brasil, 1500, 05503-900 São Paulo, Brasil. (cinquini@butantan.gov.br)

\begin{abstract}
Reproductive capacity of Bothrops jararaca (Serpentes, Viperidae). The relationship between female body size and egg number was examined in the snake Bothrops jararaca (Wied, 1924). Some authors have demonstrated that the estimated clutch size may be affected by the inclusion or exclusion of dead neonates or nondeveloped eggs. The reproductive system of 254 mature females newly arrived from nature was examined and $29.55 \%$ $(\mathrm{n}=75)$ had eggs in the oviducts in different stages of embryonic development. The presence of atresic eggs was observed in $34.5 \%$ of those females. A significant difference was observed in the number of eggs in the right and left oviducts $(\mathrm{t}=9.48, \mathrm{P}<0.001)$, but not in the number of atresic eggs $(\mathrm{t}=1.110, \mathrm{P}=0.278)$. The correlation of female body size and egg number was greater when all, the normal and atresic eggs were considered $(\mathrm{r}=0.63, \mathrm{P}<0.001)$. The exclusion of atresic eggs from the clutch size decreased slightly this correlation $(\mathrm{r}=0.54, \mathrm{P}<0.001)$.
\end{abstract}

KEYWORDS. Reproductive output, clutch size, atresic eggs, reproductive allometry.

\section{INTRODUÇÃO}

Observações sobre o tamanho da ninhada são as mais abundantes fontes de informação a respeito da reprodução em serpentes (TURNER, 1977). FARR \& GREGORY (1991) demonstraram que algumas estimativas do tamanho da prole podem ser afetadas em vários graus pela inclusão ou exclusão de filhotes mortos ou ovos não desenvolvidos. A maioria dos autores não explica como o número de neonatos foi calculado, e entre os que o fazem, não existe uma uniformidade das informações (FARR \& GREGory, 1991). Ford \& Seigel (1989) calcularam o número de descendentes como o total de filhotes vivos e natimortos, enquanto que GREGORY (1977) usou o total de animais vivos e natimortos com aspecto normal. Segundo FARR \& GREGORY (1991), todos os componentes da ninhada devem ser tabulados em estudos de reprodução de serpentes pelo fato de, até o momento, não se ter conhecimento se a ocorrência de jovens mortos, jovens em desenvolvimento incompleto ou ovos não-desenvolvidos, tem interferência do cativeiro.

A relação entre o tamanho da ninhada e o tamanho do corpo da fêmea tem sido documentada em uma ampla variedade de répteis, mostrando uma correlação significativa entre elas na maioria das espécies ( $\mathrm{FITCH}_{\text {, }}$ 1963; Bauman \& Metter, 1977; Kofron, 1983; Kihara et al., 1985; KING, 2000).

Investigações prévias sobre a serpente neotropical Bothrops jararaca (Wied,1824) relataram vários aspectos da biologia de conduta (SAZIMA,1988), relação entre o tamanho da ninhada e comprimento da fêmea e ciclo ovariano (JANEIRO-CINQUIN et al., 1990, 1993). Objetiva-se informar o número de ovos normais e atrésicos, descrever sua relação com o tamanho da fêmea e avaliar a influência da inclusão ou exclusão de ovos atrésicos nesta relação, em B. jararaca.

\section{MATERIALEMÉTODOS}

Um total de 254 fêmeas maduras de $B$. jararaca recém-chegadas da natureza, provenientes das regiões sul e sudeste do Brasil, foi examinado no Laboratório de Herpetologia do Instituto Butantan, durante os anos de 1981 e 1982. Os animais sofreram eutanásia por dióxido de carbono (CO ) e em seguida os ovos foram contados in situ por meio ${ }^{2}$ de laparotomia em ambos os ovidutos. Do total de animais analisados, 75 apresentavam ovos nos ovidutos, sendo estes utilizados neste estudo. Todos os animais foram destinados a outros projetos de pesquisa em desenvolvimento na própria instituição e, posteriormente, foram utilizados como material didático.

Os dados coletados neste estudo incluíram comprimento rostro-cloacal $(\mathrm{CRC})(\mathrm{cm})$, número de ovos normais e atrésicos em cada oviduto. Os ovos atrésicos se caracterizaram pela presença de uma massa dura de vitelo que é expelida durante o parto.

A relação entre o número de ovos normais e atrésicos e o comprimento rostro-cloacal (CRC) da fêmea foi calculada com as variáveis transformadas em logarítmico natural segundo KING (2000).

Para comparação das médias do número de ovos normais e atrésicos em ambos ovidutos, foi utilizado o teste $t$ Student pareado com nível de significância de 5\%.

\section{RESULTADOS}

Do total de 75 fêmeas de B. jararaca, 61 (81,3\%) se encontravam no início do desenvolvimento embrionário (estágios 1-8, ZEHR, 1962) e 14 (18,7\%) nos estágios finais de desenvolvimento (estágios 34-36, ZeHR, 1962). Deste total foram observadas 60 (80\%) com maior número de ovos no oviduto direito, oito $(10,7 \%)$ com igual número em ambos os ovidutos, cinco $(6,7 \%)$ com ovos somente no oviduto esquerdo e duas $(2,7 \%)$ com ovos somente no direito.

A variação do número de ovos de $B$. jararaca foi de 4 a 34 em ambos os ovidutos, com uma média de $14,2+5,86$ ovos por fêmea. O oviduto direito apresentou uma média de ovos de $9,5+4,0$ e o esquerdo $5,7+2,7$, mostrando uma diferença significativa entre eles ( $\mathrm{t}=9,48, \mathrm{gl}=74, \mathrm{P}<0,001)$.

Os ovos atrésicos estavam presentes em $34,5 \%$ das 
fêmeas, com uma média de 3,5+4,61 por fêmea, sendo $56,7 \%$ no oviduto direito e $43,3 \%$ no esquerdo. Não foi observada diferença significativa entre o número de ovos atrésicos no oviduto direito e esquerdo $(\mathrm{t}=1,110, \mathrm{gl}=25$, $\mathrm{P}=0,278$ ).

Foi observada uma diferença na correlação entre o comprimento rostro-cloacal da fêmea e o tamanho da ninhada relacionada à inclusão ou não do número de ovos atrésicos. A correlação entre o tamanho da fêmea e o número de ovos foi maior quando ovos normais e atrésicos foram considerados $(\mathrm{r}=0,63, \mathrm{P}<0,001)$ (fig. 1). A exclusão de ovos atrésicos do tamanho da ninhada diminui levemente está correlação ( $\mathrm{r}=0,54, \mathrm{P}<0,001)$ (fig. 2).

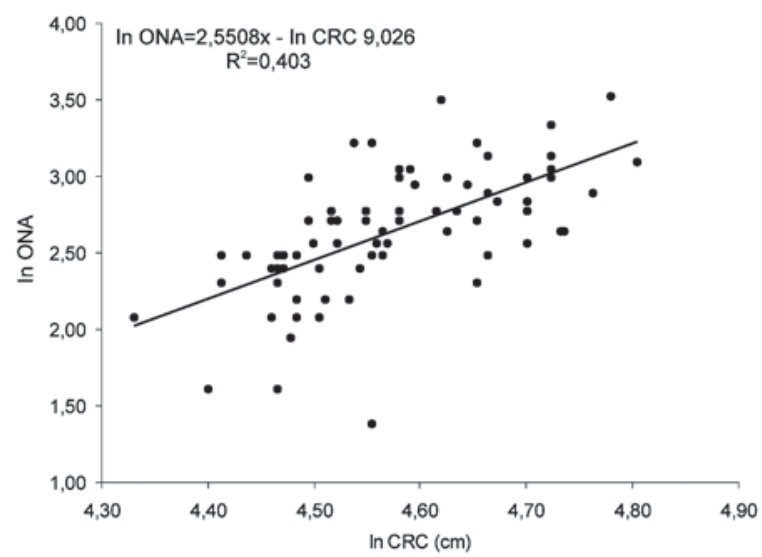

Fig. 1. Relação entre o comprimento da fêmea e o número de ovos + ovos atrésicos de Bothrops jararaca $(n=75)$, baseada nas variáveis transformadas em logaritmo natural (CRC, comprimento rostro-cloacal; ONA, ovos normais + atrésicos).

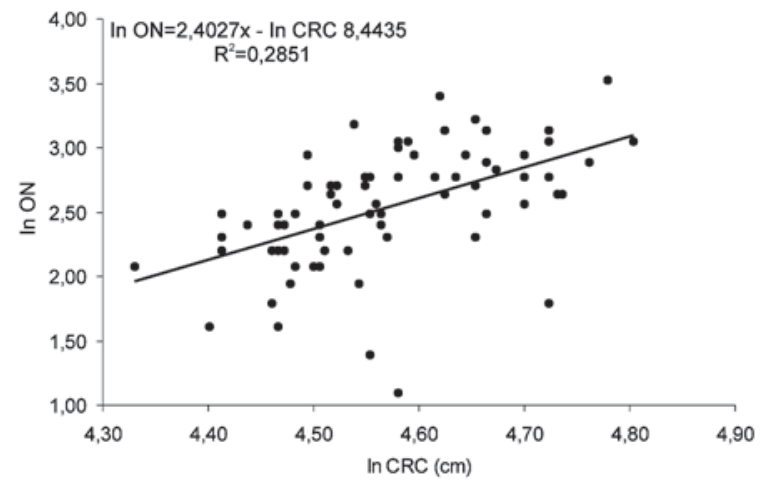

Fig. 2. Relação entre o comprimento da fêmea e o número de ovos de Bothrops jararaca $(\mathrm{n}=75)$, baseada nas variáveis transformadas em logaritmo natural (CRC, comprimento rostro-cloacal; ON, ovos normais)

\section{DISCUSSÃO}

O entendimento do potencial reprodutivo das serpentes inclui, entre outras informações, o número de filhotes por ninhada (GiBBONS, 1972). Numerosas espécies de serpentes produzem ninhadas pequenas, com um a quatro ovos ou filhotes, contudo algumas espécies podem produzir 100 ou mais ovos (FITCH, 1970). Dentre as serpentes mais prolíficas encontram-se as boas gigantes e as pítons, além de duas espécies de Viperidae, Bothrops atrox (Linnaeus, 1758) e Crotalus basiliscus (Cope,1864) (FiTcH, 1970).

A média de ovos por fêmea em Bothrops jararaca foi de 14,2, sendo este resultado similar aos encontrados por BRENo et al. (1990), com uma média anual de ovos entre 9,4 a 16,3 para esta espécie, e ArAúso (1978) com $B$. alternatus (Duméril, 1854), com uma média de 13 ovos. Em B. moojeni (Hoge, 1966), B. atrox e B. asper (Garman, 1883) o número de ovos foi maior, sendo 50, 33 e 14 a 86 respectivamente (Leloup, 1975; Hoge \& FEDERSONI, 1976/ 1977), enquanto valores menores foram encontrados para $B$. neuwiedi (Wagler, 1824) e B. jararaca mantidas em cativeiro, com uma média de 6,8 e 8,04 filhotes por ninhada, respectivamente (Alves et al., 1998, 2000).

Segundo SAzima (1992), o tamanho da ninhada de $B$. jararaca está dentro da média $(10,75)$ encontrada para a grande maioria das serpentes e se aproxima da média mais alta conhecida para viperídeos ( 2 a 16 filhotes por fêmea) (SEIGEL \& Ford, 1987).

De acordo com KовA et al. (1970), o número de ovos no oviduto direito é sempre maior que o do lado esquerdo em serpentes, devido ao posicionamento mais anterior daquele em relação ao esquerdo. Em Vipera berus (Linnaeus, 1758), 61,8\% dos embriões estavam presentes no oviduto direito e 38,2\% no esquerdo, e em Agkistrodon piscivorus (Lacèpéde, 1789), 60,7\% estavam no lado direito e 39,3\% no esquerdo (Volsoe, 1944; Wharton, 1966). Em $B$. jararaca foi observado um número maior de ovos no oviduto direito $(69,92 \%)$ quando comparado com o esquerdo $(30,08 \%)$, sendo estas porcentagens similares às observadas nas espécies acima mencionadas. Esta assimetria do oviduto em serpentes permite que grande parte ou todos os ovos do oviduto esquerdo eclodam antes que os ovos do oviduto direito, por serem em menor número (BLACKBURN, 1998).

Alves et al. (2000), estudando o aspecto reprodutivo de $B$. jararaca em cativeiro observaram que do total de ovos paridos (515), 67 eram atrésicos $(13,12 \%)$. Os dados apresentados para $B$. jararaca recém-chegadas da natureza mostraram que do total de ovos analisados, 7,89\% eram atrésicos. Alves et al. (1998), relataram diferença no número de ovos atrésicos em $B$. neuwiedi mantidas em cativeiro $(28,46 \%)$ e recém-chegadas da natureza $(22,96 \%)$.

Em alguns colubrídeos, viperídeos (AldRIDGE, 1979, 1982) e elapídeos (SHINE, 1977) foi observada correlação entre o tamanho da ninhada e o comprimento rostro-cloacal da fêmea. SOLÓRZANO \& CERDAS (1987, 1989), estudando a biologia reprodutiva de Crotalus durissus durissus (Linnaeus, 1758) e de B. asper da Costa Rica, encontraram uma correlação significativa entre o comprimento total das fêmeas e o número de neonatos $(\mathrm{r}=0,58$ e $\mathrm{r}=0,78$, respectivamente $)$. Correlação significativa também foi constatada por JANEIRO-CINQUINI et al. (1990) entre o comprimento da mãe e o número de fillhotes $(\mathrm{r}=$ 0,45 ) em uma outra população de serpentes $B$. jararaca.

FARR \& GREGORY (1991), ao estudarem Thamnophis elegans (BAIRD \& GIRARDI, 1853), observaram que a inclusão ou não de determinados componentes (filhotes mortos ou ovos não desenvolvidos) alterava a intensidade da correlação entre o tamanho da ninhada e o comprimento 
da mãe. Os resultados com $B$. jararaca mostraram uma correlação positiva significativa entre o comprimento da mãe e o tamanho da ninhada. A intensidade desta correlação foi afetada pela inclusão ou não de ovos atrésicos. Quando o número de ovos atrésicos foi retirado da estimativa do tamanho da prole, a correlação foi menor. Segundo FARR \& GREGORY (1991), é interessante citarmos como a estimativa do tamanho da ninhada foi calculada, pois dependendo do objetivo da investigação, pode interferir na avaliação dos dados.

A determinação do tamanho da ninhada é vantajosa nestes animais para comparação entre populações, especialmente se alguns dados são baseados na contagem derivada da palpação de fêmeas grávidas ou de dissecação de serpentes no início da gestação (FARR \& GREGORY, 1991).

Agradecimentos. Aos pesquisadores do Laboratório de Herpetologia do Instituto Butantan, Eliana O. Serapicos e Hana Suzuki pela leitura crítica do manuscrito e ao Sávio S. Sant'Ana pelo auxílio na análise dos dados estatísticos.

\section{REFERÊNCIAS BIBLIOGRÁFICAS}

AldRIDGE, R. D. 1979. Female reproductive cycles in the snakes Arizona elegans and Crotalus viridis. Herpetologica, Chicago, (35):256-261.

1982. The ovarian cycle of the watersnake Nerodia sipedon and effects of hypohysectomy and gonadotropin administration. Herpetologica, Chicago, (38):71-79.

Alves, M. L.; Araúso, M. L. DE et al. 1998. Atividade reprodutiva de Bothrops neuwiedi em cativeiro (Serpentes, Viperidae). Iheringia, Série Zoologia, Porto Alegre, (84):185-191.

2000. Aspectos da biologia reprodutiva de Bothrops jararaca em cativeiro (Serpentes, Viperidae). Iheringia, Série Zoologia, Porto Alegre, (89):187-192.

AraúJo, M. L. DE. 1978. Notas sobre ovos de serpentes (Boidae, Colubridae, Elapidae e Viperidae). Iheringia, Série Zoologia, Porto Alegre, (51):9-37.

Bauman, M. A. \& Metter, D. E. 1977. Reproductive cycle of the northern watersnake, Natrix s. sipedon (Reptilia, Serpentes, Colubridae). Journal of Herpetology, Kansas, (11):51-59.

BlackBurn, D. G. 1998. Structure, function, and evolution of the oviducts of squamate reptiles with special reference to viviparity and placentation. Journal of Experimental Zoology, Philadelphia, (282):560-617.

Breno, M. C.; Yamanouye, N. et al. 1990. Maintenance of the snake Bothrops jararaca (Wied, 1824) in captivity. The Snake, Nugata (22):126-130

FARR, D. R. \& GREgory, P. T. 1991. Sources of variation in estimating litter characteristics of the garter snake, Thamnophis elegans. Journal of Herpetology, Kansas, (25):261-268.

Fітсн, H. S. 1963. Natural history of the racer, Coluber constrictor. University of Kansas Museum of Natural History Miscellaneous Publication, Lawrence, (15):351-468. 1970. Reproductive cycles of lizards and snakes. University of Kansas Museum of Natural History Miscellaneous Publication, Lawrence, (52):1-247.

Ford, N. B. \& SeIgel, R. A. 1989. Relationships among body size, and egg size in three species of oviparous snakes. Herpetologica, Chicago, (45):75-83.
GibBons, J. W. 1972. Reproduction, growth, and sexual dimorphism in the canebrake rattlesnake (Crotalus horridus attricaudatus). Copeia, Lawrence, 1972:222-226.

Gregory, P. T. 1977. Life-history parameters of the red-sided garter snake (Thamnophis sirtalis parietalis) in an extreme environment the Interlake District of Manitoba. Natural Museum Canadian Publication Zoology, Ottawa, (13):144.

Hoge, A. R. \& Federsoni JR., P. A. 1976/1977. Observações sobre uma ninhada de Bothrops atrox (Linnaeus, 1758) (Serpentes: Viperidae: Crotalinae). Memórias do Instituto Butantan, São Paulo, (40/41):19-36.

JANEIRo-Cinquini, T. R. F.; Leinz, F. F. et al. 1990. Body size-litter relationships and some characteristics of litters in Bothrops jararaca. Bulletin of the Chicago Herpetological Society, Chicago, 25(5):84-85.

1993. Ovarian cycle of the snake Bothrops jararaca. Memórias do Instituro Butantan, São Paulo, 55(1):33-36.

Kinara, H.; Hashimura, S. et al. 1985. Sex composition and reproduction of habu, Trimeresurus flavoviridis, the venomous snake. The Snake, Nugata, (17):135-139.

KING, R. B. 2000. Analyzing the relationship between clutch size and female body size in reptiles. Journal of Herpetology, Kansas, 34(1):148-150.

Кова, K.; Мовiмото, H. et al. 1970. Eggs and egg laying in the habu, Trimeresurus flavoviridis of Amani-oshima Island. The Snake, Nugata, (2):22-31.

Kofron, C. P. 1983. Female reproductive cycle of the Neotropical snail-eating snake Sibon sanniola in Northern Yucatan, México. Copeia, Lawrence, (4):963-969.

Leloup, P. 1975. Observation sur la reproduction de Bothrops moojeni Hoge en captivité. Acta Zoology et Pathologyca Antverpiensia, Antverpen, (62):173-201.

SazimA, I. 1988. Um estudo de biologia comportamental de jararaca, Bothrops jararaca, com uso de marcas naturais. Memórias do Instituto Butantan, São Paulo, 50(3): 83-99. 1992. Natural history of the jararaca pitviper, Bothrops jararaca, in Southeastern Brazil. In: Campbel, J. A. \&. Brodie, E. D. Jr. eds. Biology of the Pitvipers, Texas, Selva Tyler. p. 199-216.

Seigel, R. A. \& Ford, N. B. 1987. Reproductive ecology. In: Seigel, R. A.; Collins, J. T. \& Novak, S. S. eds. Snakes: ecology and evolutionary biology. New York, Mcmillan. p. 210-252.

Shine, R. 1977. Reproduction in Australian elapid snakes II. Female reproductive cycles. Australian of Journal Zoology, Melbourne, (25):655-666.

SolóRzano, A. \& Cerdas, L. 1987. Biologia reproductiva de la cascabel centroamericana Crotalus durissus durissus (Serpentes: Viperidae) en Costa Rica. Revista de Biologia Tropical, San Jose, (36):221-226.

1989. Reproductive biology and distribution of the terciopelo, Bothrops asper Garman (Serpentes: Viperidae) in Costa Rica. Herpetologica, Chicago, 45(4):444-450.

Turner, F. B. 1977. The dynamics of populations of squamates and crocodilians. In: Gans, C. \& TinkLE, D. W. eds. Biology of Reptilia, New York, Academic. v. 7, p. 157-264.

Volsoe, H. 1944. Structure and seasonal variation of the male reproductive organs of Vipera berus (L.). Spolia Zoolology Museum Hauniensis, Copenhagen, (5):1-172.

Wharton, C. H. 1966. Reproduction and growth in the cottonmouth Agkistrodon piscivorus Lacèpéde of Cedar Key, Florida. Copeia, Lawrence,1966(2):149-161.

Zehr, D. R. 1962. Stages in the normal development of the common garter snake, Thamnophis sirtalis sirtalis. Copeia, Lawrence, 1962:322-329. 